\title{
Meta
}

Journal des traducteurs

Translators' Journal

\section{ASOBELE-TIMoTHy, Samuel Jide (2016) : Translation Studies in Africa. Lagos: Promocomms Ltd., 599 p.}

\section{Segun Afolabi}

Volume 63, numéro 1, avril 2018

URI : https://id.erudit.org/iderudit/1050527ar

DOI : https://doi.org/10.7202/1050527ar

Aller au sommaire du numéro

Éditeur(s)

Les Presses de l’Université de Montréal

ISSN

0026-0452 (imprimé)

1492-1421 (numérique)

Découvrir la revue

Citer ce compte rendu

Afolabi, S. (2018). Compte rendu de [Asobele-Timothy, Samuel Jide (2016) :

Translation Studies in Africa. Lagos : Promocomms Ltd., 599 p.] Meta, 63(1),

260-263. https://doi.org/10.7202/1050527ar

Ce document est protégé par la loi sur le droit d'auteur. L'utilisation des services d'Érudit (y compris la reproduction) est assujettie à sa politique d'utilisation que vous pouvez consulter en ligne.

https://apropos.erudit.org/fr/usagers/politique-dutilisation/
Cet article est diffusé et préservé par Érudit.

Érudit est un consortium interuniversitaire sans but lucratif composé de l’Université de Montréal, l'Université Laval et l'Université du Québec à Montréal. Il a pour mission la promotion et la valorisation de la recherche. https://www.erudit.org/fr/ 
Cognitive aspects of community interpreting: Toward a process model by Englund Dimitrova and Tiselius provides an overview of research into community interpreting, in particular the two aspects of the community interpreter's role, namely the interpreting part and the managing of the interaction of the encounter. The interpreter, therefore, cannot be studied in isolation but rather in terms of the behaviour of all participants, so there is relatively little on the cognitive aspects of interpreting and that is largely on simultaneous interpreting.

The authors propose the concept of monitoring to capture how aspects of interaction are managed by the community interpreter. In addition to the monitoring that occurs in all speakers as evidenced by repairs and corrections, community interpreters monitor their comprehension of the primary party's utterance, the relation of that utterance to the last part that was interpreted to see if it was correctly understood, and their own memory and processing capacity to see if they need to take the turn. And in speaking they monitor their own utterances in the language in question, the relation of their utterance to the primary party's utterance, and the verbal and non-verbal reactions of the primary parties.

Community interpreters not only monitor in relation to primary parties' previous utterances and reactions, they plan ahead of interactions and coordinate turn-taking. The authors also suggest that professional self-concept is critical in the community interpreting process. They illustrate this with excerpts from two interpreted encounters, one with an interpreting student, whose professional self-concept is not well enough developed to give him confidence to take an active role in managing turn-taking, and a certified professional interpreter with eight or nine years of experience who handles turn-taking well. The authors suggest that video recordings are necessary to study cognitive aspects of community interpreting as gaze patterns and gestures are important.

CANDACE SÉGuinot York University, Toronto, Canada

\section{REFERENCES}

Abdallah, Kristiina (2012): Translators in Production Networks. Reflections on Agency, Quality and Ethics. Joensuu: University of Eastern Finland.

Newell, Allen and Simon, Herbert A. (1961): Computer simulation of human thinking. A theory of problem solving expressed as a computer program permits simulation of thinking processes. Science. 134(3495):2011-2017.

Wheeler, Michael (2005): Reconstructing the Cognitive World. The Next Step. Cambridge: MIT Press.
Asobele-Timothy, Samuel Jide (2016): Translation Studies in Africa. Lagos: Promocomms Ltd., 599 p.

Malgré l'abondante documentation scientifique existant en traductologie dans le monde, il est assez rare de trouver des ouvrages traitant de la discipline dans le contexte africain. Très peu de chercheurs, en effet, se sont penchés sur l'histoire et l'état des lieux de la traduction en Afrique. Parmi ceux-ci, nous pouvons citer Bandia (2001/2005) et Simpson (1985). C’est précisément ce manque de textes de référence qui est à l'origine de la parution de Translation Studies in Africa (p. 83-84). Il convient de signaler d'emblée que l'ouvrage est une collection de réflexions d'Asobele ${ }^{1}$ sur la traduction professionnelle et surtout la traduction littéraire en Afrique.

Le livre s'ouvre sur un avant-propos par Olaoye Abioye, traducteur littéraire chevronné et professeur titulaire retraité à l'Université de Lagos, qui n'hésite pas à recommander Translation Studies in Africa à tout étudiant et enseignant de traduction. Ensuite viennent les pages de dédicace, de remerciements et d'introduction. Les 36 sous-titres ou chapitres que contient le livre peuvent être facilement scindés en trois grandes rubriques: la pédagogie de la traduction (4 chapitres), la recherche traductologique (4 chapitres) et la traduction littéraire (28 chapitres). L'ouvrage se termine sur une conclusion et des références bibliographiques.

Dans l'introduction, intitulée «Multilinguisme et mondialisation en cours: quel rôle pour les écoles de traduction et d'interprétation en Afrique?», Asobele lance le débat au moyen d'un proverbe africain d'origine yoruba ${ }^{2}$ qui dit: «Si un enfant n'a jamais quitté la ferme de son père, pour se rendre en visite dans la ferme d'autres fermiers, il croira toujours et avec raison que la ferme de son père est la plus grande du monde» (p. vii). Par ce proverbe, Asobele informe son lecteur que c'est lorsqu'il a quitté son pays natal pour un séjour de recherche au Département de linguistique et de traduction de l'Université de Montréal, en 1998, que ses yeux ont été ouverts à l'immense réalité de la mondialisation, aux enjeux du multilinguisme et au rôle important que joue la traduction dans ces phénomènes à la fois complexes et incontournables. Selon l'auteur, ce sont effectivement ses découvertes et ses expériences à Montréal qui ont donné naissance à Translation Studies in Africa. L'introduction souligne donc le rôle de la traduction comme moteur de la mondialisation en cours, et donne au lecteur un aperçu de ce à quoi il doit s'attendre dans le reste du livre.

Dans le premier chapitre, «Integration in Africa: The need for translation schools», Aso- 
bele présente un survol historique de la mise en place du programme de master en traduction (Master of Arts in Translation and Interpretation) de l'Université de Lagos, au Nigéria. Selon lui, bien avant la création le 10 juin 1975 de la Communauté économique des États de l'Afrique de l'Ouest (CEDEAO), une organisation visant l'intégration socio-économique des pays de la sous-région, les recommandations du Comité d'experts de l'Organisation de l'unité africaine (aujourd'hui l'Union africaine) favorisaient entre autres le bilinguisme anglais-français, comme une issue à la véritable malédiction de Babel dans laquelle sont impliquées les plus de 1200 langues du continent africain (p. 1). Il s'agissait là d'un appel à la création d'écoles de traduction en Afrique. Ensuite, la tenue du festival culturel panafricain Festival of Arts and Culture (FESTAC) à Lagos, en janvier-février 1977, a accentué le besoin de former des traducteurs et des interprètes, car le volume de travail à réaliser pendant ce festival d'envergure internationale dépassait largement le nombre des personnes capables de l'exécuter. Les langues de travail du festival étaient le français, l'anglais, l'espagnol, l'arabe et le portugais. C'est ainsi qu'Ekundayo Simpson ${ }^{3}$, alors directeur des services de traduction au Secrétariat du FESTAC, a conçu l'idée de lancer le programme de master professionnel en traduction et en interprétation à l'Université de Lagos (UNILAG). Ce programme n'a pourtant démarré qu'en 1992, dix ans après le départ à la retraite de son initiateur (Asobele 1999: iv). Dans le reste du chapitre, l'ensemble du programme de master en traduction de l'UNILAG (objectifs, avantages, conditions d'admission, cours offerts et leur description, corps enseignant, stage, etc.) est présenté en détail ${ }^{4}$.

Le deuxième chapitre, "Integration in West Africa: The need for translation Schools", s'enchaîne de manière logique avec le chapitre précédent pour mettre en lumière le concept de l'intégration en le situant dans le contexte précis de la CEDEAO. Le chapitre traite donc des considérations sociale, politique et économique de l'intégration. L'intégration y est perçue comme le processus de conjugaison d'intérêts et d'efforts au profit d'une organisation ou d'une institution plus grande, et pour le bien-être des éléments la constituant. Pour atteindre ces objectifs, la traduction a un rôle indéniable à jouer, surtout dans une communauté linguistiquement diversifiée comme l'Afrique. Par ailleurs, afin de mieux comprendre les enjeux de l'intégration au sein de la CEDEAO, Asobele dresse un panorama de l'histoire et de l'influence de la colonisation sur les pays francophones de la sousrégion ouest-africaine. Il traite ensuite des efforts unificateurs de la CEDEAO pour rompre avec ce passé et intégrer toute la sous-région en une seule communauté. Encore une fois, l'importance des écoles de traduction et d'interprétation pour ce faire est mise en exergue.

Dans les quatrième et cinquième chapitres, Asobele fait une sorte de retour en arrière sur le programme de master en traduction de l'Université de Lagos, en y traitant des sujets suivants: "The pedagogy of translation at Lagos School of Translation. Projects and memoires in translation and final year results» et "Lagos School of translation and interpretation: the ambivalence and ambiguity of "A" language in Africa.» On lit dans le chapitre quatre le rapport sur le système d'évaluation (examens et notation) ainsi que les sujets de mémoires de fin de programme préparés par les étudiants au master en traduction de l'UNILAG entre 1992 et 2003. Plus particulièrement, l'auteur fait mention des modifications qui ont été proposées à ce programme en vue de tenir compte des langues nigérianes comme la langue « $\mathrm{A}$ » des apprentis traducteurs. Cette proposition d'amendement s'associe à la campagne pour la promotion des langues maternelles africaines menée par des pédagogues et linguistes africains, dont Babatunde Fafunwa, ancien ministre de l'Éducation fédérale du Nigéria. Comme le signale Asobele, «it is the realisation of the importance of language " $\mathrm{A}$ " in the training of Nigeria's professional translators and interpreters that encouraged us to recommend Education in mother tongue by Prof Babs Fafunwa to our students» (p. 60). Dans le même ordre d'idées, le chapitre cinq traite davantage de l'ambiguïté de la langue « $\mathrm{A}$ » dans le contexte des traducteurs et interprètes africains. Pour Asobele, la langue « $\mathrm{A}$ » des traducteurs et interprètes africains sera la langue maternelle de ceux-ci, par exemple, le yoruba pour un traducteur nigérian de la tribu yoruba et le wolof pour son homologue sénégalais. Il remarque: "This is one of the reasons why we feel that the Lagos School should show the light and give pride of place to African languages in its programs at the dawn of $21^{\text {st }}$ Century» (p. 82).

Du sixième au neuvième chapitre, que nous classons sous la rubrique de recherche traductologique, il est fait état des différents travaux réalisés par les chercheurs ainsi que les associations professionnelles et savantes pour la promotion de la traductologie dans le monde et plus spécifiquement en Afrique. Dans la partie intitulée «The importance of translation journals in the training of translation professionals: the debate on "A" language for Africa", Asobele met en évidence les contributions de la revue Meta au développement de la traductologie en tant que discipline. Â l'instar de Meta, d'autres revues entièrement consacrées à la recherche traductologique citées par Asobele sont Babel, Le Linguiste, Van Taal Tot Tal, Hieronymus et Traduire (toutes en Europe). Ensuite, 
l'auteur cite des revues similaires en Inde telles que International Journal of Translation (IJT) et Translation Today, dont un bref compte rendu de l'édition inaugurale (2004) est présenté (p. 113-124). Il convient de noter qu'en Afrique, une seule revue semblable figure sur la liste, il s'agit de Turjuma, la revue de la King Fahd School of Translation à Tanger, Maroc, qui a fait une nouvelle apparition en 1992. Pour conclure le chapitre, l'auteur rappelle qu'en 1999, Eureka - a journal of humanistics studies, revue publiée par le Département de langues européennes de l'Université de Lagos, a consacré un de ses numéros à la traduction. Nous pouvons retenir de cette présentation sommaire des revues consacrées à la traductologie que le continent africain accuse encore du retard comparativement au reste du monde.

La rubrique continue sur « Keynote Address at the 8th Congress and Workshop of the Nigerian Institute of Translators and Interpreters ». Ce discours liminaire intitulé «The Training of Translators and Interpreters in West Africa Today and Tomorrow» a été prononcé par Ekundayo Simpson qui met l'accent sur la nécessité pour les pays ouest-africains de conjuguer les efforts afin de promouvoir la formation des traducteurs et interprètes. Cette partie se termine sur l'article "Translation and the transfer of knowledge and technical know-how in the year 2000 and beyond ", dans lequel Asobele insiste à nouveau sur l'importance des revues consacrées à la traduction. Il propose également la traduction d'articles issus des revues scientifiques spécialisées dans le but de faciliter le transfert du savoir dans le monde entier. Dans sa conclusion, l'auteur souligne l'apport de la TAO (traduction assistée par ordinateur).

Les 28 chapitres figurant dans la dernière rubrique de Translation Studies in Africa sont essentiellement consacrés à la traduction littéraire, ses enjeux et défis. C'est la dynamique de la traduction littéraire que les articles dans cette partie cherchent à souligner, ce qui est lucidement démontré par l'analyse critique d'un certain nombre de textes littéraires traduits. Le message central des articles de cette section porte sur l'utilité de la traduction littéraire et de la littérature comparée. Voici comment le présente Asobele:

The utilitarian significance of literary translation and comparative literature is that they make scholars, writers, translators and critics the world over to pay a particular attention to the literatures of the countries whose works are translated into several world languages. (p. 217)

Pour illustrer cette prise de position, l'auteur inclut une longue liste d'ouvrages en langues européennes traduits en yoruba (Annexe I), d'ouvrages yoruba traduits en français (Annexe II), d'ouvrages en yoruba traduits en anglais (Annexe III), d'ouvrages d'auteurs francophones africains traduits en anglais (Annexe IV) et d'ouvrages d'auteurs anglophones africains traduits en français (Annexe V) (p. 173-180; 198-206). Parmi ces œuvres traduites, nous citerons notamment celles de Wole Soyinka, un écrivain nigérian et lauréat du prix Nobel de littérature (1986), ainsi que de Sembene Ousmane, un romancier-cinéaste renommé du Sénégal. Le reste de cette partie présente des critiques rédigées par Asobele sur quelques-unes de ces traductions où il soulève certains problèmes pointus de la traduction littéraire, particulièrement en ce qui a trait à la culture et au contexte africains.

En guise de conclusion, Translation Studies in Africa se veut un livre de référence important sur la traductologie, ses activités et son développement sur le continent africain. La qualité éditoriale de l'ouvrage laisse toutefois à désirer, car on y remarque ci et là des coquilles ${ }^{5}$. En dépit de cela, nous ne pouvons que recommander la lecture de Translation Studies in Africa à toutes celles et tous ceux qui s'intéressent à la traduction en Afrique, qu'ils soient traducteurs, interprètes, étudiants, enseignants ou chercheurs. Par ailleurs, comme le fait remarquer Asobele dans la conclusion, tout n'est pas encore dit à propos de la traductologie en Afrique; d'énormes champs de recherche intéressants, tels que l'interprétation de conférence, l'interprétation communautaire, la formation des interprètes et des traducteurs professionnels, restent à explorer et à documenter aux fins de références futures (p. 565-567).

Segun Afolabi Université Laval, Québec, Canado

\section{NOTES}

1. L'auteur de l'ouvrage, S. J. Timothy-Asobele, est professeur titulaire et ancien chef du Département de langues européennes de l'Université de Lagos au Nigéria. Il a été aussi, pendant de longues années, coordinateur du programme de Master en traduction de la même université, programme auquel il fait amplement allusion dans le livre.

2. Les Yoruba constituent un groupe ethnique majeur du Nigéria. Ils sont concentrés au sudouest du pays.

3. Ekundayo Simpson, professeur titulaire retraité de l'Université de Lagos est un ancien étudiant de l'Université Laval, Québec, où il a préparé une thèse de doctorat sous la direction de Jean Darbelnet, en 1972.

4. Force est de signaler que présentement, le programme n'existe que sur papier, étant donné que la dernière promotion d'étudiants admis au programme date de l'année scolaire $2003 / 2004$. 
5. Par exemple, Jean Vinay est écrit par erreur au lieu de Jean Darbelnet (p. 57, ligne 5); translation au lieu de translators (p. 62, avant-dernier paragraphe); on voit la répétition du contenu des pages 74 à 87 dans les pages 96 à 109; de nombreuses fautes de frappe (p. 129, 132, 144, $145,146,154,173,174,409)$, pour ne citer que celles-là. Nous espérons que l'auteur aura l'occasion de les corriger dans une prochaine édition.

\section{RÉFÉRENCES}

Asobele, Timothy (1999): New Perspectives in the Training of Translators and Interpreters in Nigeria. Lagos: Printview Publishers.

Bandia, Paul (2005): Esquisse d'une histoire de la traduction en Afrique. Meta. 50(3):957-971.

Bandia, Paul (2001): African Tradition. In: Mona BAKER (dir.). Routledge Encyclopedia of Translation Studies. New York: Routledge, 295-305.

Simpson, Ekundayo (2007a): Translation Interventions: Papers and Addresses on Translation, Language, Literature and Culture. Lagos/ Abuja: Interlingua Limited.

Simpson, Ekundayo (2007b): Translation: Principles and Applications. Lagos/Abuja: Interlingua Limited.

Simpson, Ekundayo (1985): Translation Problems of African Countries. In: Hildegund BüHLER (dir.). Translators and Their Position in Society. Proceedings of the 10th World Congress of FIT. Vienne: Braumüller, 105-110.

Pragana Dantas, Marta et Henriques Pereira De SouzA, Germana (dir.) (2016): A tradução de obras francesas no Brasil: trajetórias, debates, deslocamentos. Campinas: Pontes Editores, $184 \mathrm{p}$.

Ce volume du recueil Histórias da Tradução regroupe les interventions survenues au cours de la III ${ }^{e}$ Rencontre des traducteurs d'œuvres littéraires françaises au Brésil, organisée conjointement par le Programme de spécialisation en lettres et la Coordination du programme de baccalauréat en traduction de l'Université fédérale de la Paraíba, qui a eu lieu en octobre 2014 à João Pessoa. Ce livre est la suite des recueils précédents organisés à l'occasion des $\mathrm{I}^{\text {re }}$ et $\mathrm{II}^{\mathrm{e}}$ Rencontres des traducteurs d'œuvres littéraires françaises, qui ont eu lieu respectivement en 2006 et 2009 . Il ressort des articles qui composent ce recueil que les littératures nationales, par le biais de la traduction, entrent en compétition avec d'autres traductions et modèles, de manière directe ou implicite, dans l'analyse des œuvres françaises traduites au Brésil.

Le recueil débute par une réflexion à propos des inégalités et des asymétries qui régissent la circulation internationale de la littérature. Dans Éléments pour une cartographie de la littérature québécoise au Brésil: textes, parcours, acteurs ${ }^{1}$, Marc Charron se penche sur deux romans québécois, Une histoire américaine ${ }^{2}$ de Jacques Godbout et $R u^{3}$ de Kim Thúy. L'auteur remet en question le rôle centralisateur de la France dans la circulation des littératures en français, y compris dans la littérature québécoise, en abordant la pertinence de faire coïncider la discussion sur la traduction dans l'espace latino-américain et la problématisation de l'américanité partagée.

En revanche, dans Transpiration et récréation poétiques: une réflexion critique sur la traduction brésilienne d' "Exercice de style» de Raymond Que$n e a u^{4}$, Lia Araújo, M. de Lima et Germana Henriques Pereira de Sousa examinent les choix faits par Luiz Rezende dans la traduction brésilienne d'Exercices de style ${ }^{5}$ de Queneau. Les auteures, évoquant l'éthique dans le processus de la traduction, invitent les lecteurs à des questionnements théoriques sur les choix du traducteur quant à la visibilité de l'Autre et la traduction ethnocentrique.

Marie-Hélène C. Torres établit, dans Le pouvoir anthropophagique du traducteur: entre tradition et innovation ${ }^{6}$, un dialogue avec plusieurs théoriciens de la traduction. Elle problématise la figure du traducteur comme anthropophage, celui qui révèle ou annexe l'étranger, et met en regard les stratégies de traduction et la question de l'invisibilité. L'auteure présente deux questions fondamentales: l'agentivité du traducteur à travers ses stratégies et la lutte consciente, ou non, pour la visibilité ou la reconnaissance internationale d'une littérature.

Elle-même traductrice, Ivone C. Benedetti présente dans Traduction des sciences humaines: quelques questions à propos de la formation des traducteurs $^{7}$ une réflexion sur la préparation du traducteur au marché du travail. En mettant l'accent sur la formation dans les humanités françaises au Brésil, le texte reprend l'historique des transformations survenues au cours des cinquante dernières années dans le système d'éducation brésilien. La philosophie a été éliminée du cursus et l'histoire a perdu son statut de matière critique. Benedetti analyse les conséquences de ces lacunes pour la formation du futur traducteur qui travaille dans le domaine des sciences humaines.

Dans Les réserves de la traductrice: traduire d'autres domaines avec la psychanalyse ${ }^{8}$, Márcia Atalla Pietroluongo narre l'expérience d'une traductrice officielle au Brésil devant traduire le verbe réserver, dans le cadre d'une décision de justice en français. À partir de son histoire, il est possible de suivre les hésitations, les angoisses et les prises de décision qu'implique la traduction, surtout dans un texte juridique ayant un impact réel sur 